\title{
Rotationally invariant multipartite states
}

\author{
Dariusz Chruściński and Andrzej Kossakowski \\ Institute of Physics, Nicolaus Copernicus University, \\ Grudziądzka 5/7, 87-100 Toruń, Poland
}

\begin{abstract}
We construct a class of multipartite states possessing rotational $\mathrm{SO}(3)$ symmetry - these are states of $K$ spin- $j_{A}$ particles and $K$ spin- $j_{B}$ particles. The construction of symmetric states follows our two recent papers devoted to unitary and orthogonal multipartite symmetry. We study basic properties of multipartite $\mathrm{SO}(3)$ symmetric states: separability criteria and multi-PPT conditions.
\end{abstract}

\section{Introduction}

Symmetry plays a prominent role in modern physics. In many cases it enables one to simplify the analysis of the corresponding problems and very often it leads to much deeper understanding and the most elegant mathematical formulation of the corresponding physical theory. In Quantum Information Theory [1] the very idea of symmetry was first applied by Werner [2] to construct a highly symmetric family of bipartite $d \otimes d$ states which are invariant under the following local unitary operations

$$
\rho \longrightarrow U \otimes U \rho(U \otimes U)^{\dagger}
$$

where $U$ are unitary operators from $U(d)$ - the group of unitary $d \times d$ matrices. Another family of symmetric states (so called isotropic states [3]) is governed by the following invariance rule

$$
\rho \longrightarrow U \otimes \bar{U} \rho(U \otimes \bar{U})^{\dagger}
$$

where $\bar{U}$ is the complex conjugate of $U$ in some basis. Other symmetry groups (subgroups of $U(d)$ ) were first considered in [4].

Let us observe that the problem of symmetric bipartite states may be formulated in more general setting. Consider the composite system living in $\mathcal{H}_{\text {total }}=\mathcal{H}_{A} \otimes \mathcal{H}_{B}$ and let $G$ be a symmetry group in question. Let $\mathfrak{D}^{(A)}$ and $\mathfrak{D}^{(B)}$ denote irreducible unitary representations of $G$ in $\mathcal{H}_{A}$ and $\mathcal{H}_{B}$, respectively. Now, a state $\rho$ of the composite is Werner-like $\mathfrak{D}^{(A)} \otimes \mathfrak{D}^{(B)}$-invariant iff

$$
\left[\mathfrak{D}^{(A)}(g) \otimes \mathfrak{D}^{(B)}(g), \rho\right]=0,
$$

for all elements $g \in G$. Similarly, $\rho$ is isotropic-like $\mathfrak{D}^{(A)} \otimes \overline{\mathfrak{D}^{(B)}}$-invariant iff

$$
\left[\mathfrak{D}^{(A)}(g) \otimes \overline{\mathfrak{D}^{(B)}(g)}, \rho\right]=0 .
$$

It is clear that taking $\mathcal{H}_{A}=\mathcal{H}_{B}=\mathbb{C}^{d}$ and $\mathfrak{D}^{(A)}=\mathfrak{D}^{(B)} \equiv \mathfrak{D}$ the defining representation of $G=U(d)$ one obtains the standard Werner state [2]. Taking as $G$ a rotational group $\mathrm{SO}(3)$ one constructs a 
family of rotationally invariant states considered recently in [4] and in more details in $[5,6,7,8]$ (see also [9]). Rotationally invariant bipartite states arise from thermal equilibrium states of lowdimensional spin systems with a rotationally invariant Hamiltonian by tracing out all degrees of freedom but those two spins. Entanglement in generic spin models has recently been studied in $[10,11,12,13]$. Rotationally invariant states were recently applied in quantum optics to describe multiphoton entangled states produced by parametric down-conversion [14] (see also [15]).

In a present paper we consider a multipartite generalization of $\mathrm{SO}(3)$-invariant states. Symmetric multipartite states were first considered in [16] (see also [17]) for $G=U(d)$ and $G=O(d)$. An $N$-partite generalization of Werner state in $H_{\text {total }}=\left(\mathbb{C}^{d}\right)^{\otimes N}$ is defined by the following requirement [16]:

$$
\left[U^{\otimes N}, \rho\right]=0
$$

for all $U \in U(d)$. This definition may be slightly generalized as follows: an $N$-partite state $\rho$ living in $\mathcal{H}_{\text {total }}=\mathcal{H}_{1} \otimes \ldots \otimes \mathcal{H}_{N}$ is invariant under $\mathfrak{D}^{(1)} \otimes \ldots \otimes \mathfrak{D}^{(N)}$, where $\mathfrak{D}^{(k)}$ denotes an irreducible representation of the symmetry group $G$ in $\mathcal{H}_{k}$, iff

$$
\left[\mathfrak{D}^{(1)}(g) \otimes \ldots \otimes \mathfrak{D}^{(N)}(g), \rho\right]=0
$$

for all $g \in G$.

Recently $[18,19]$ we proposed another family of multipartite symmetric states. Our construction works for even number of parties. Consider $K$ copies of $\mathcal{H}_{A}$ and $K$ copies of $\mathcal{H}_{B}$. Let $\mathfrak{D}^{(A)}$ and $\mathfrak{D}^{(B)}$ denote irreducible unitary representations of $G$ in $\mathcal{H}_{A}$ and $\mathcal{H}_{B}$, respectively. Now, a $2 K$-partite state $\rho$ is $\left(\mathfrak{D}^{(A)} \otimes \ldots \otimes \mathfrak{D}^{(A)}\right) \otimes\left(\mathfrak{D}^{(B)} \otimes \ldots \otimes \mathfrak{D}^{(B)}\right)$-invariant iff

$$
\left[\mathfrak{D}^{(A)}\left(g_{1}\right) \otimes \ldots \otimes \mathfrak{D}^{(A)}\left(g_{K}\right) \otimes \mathfrak{D}^{(B)}\left(g_{1}\right) \otimes \ldots \otimes \mathfrak{D}^{(B)}\left(g_{K}\right), \rho\right]=0
$$

for all $\left(g_{1}, \ldots, g_{K}\right) \in G \times \ldots \times G$. Note the crucial difference between these two definitions (1.5) and (1.7): the first one uses only one element $g$ from $G$ whereas the second one uses $K$ different elements $g_{1}, \ldots, g_{K}$, and hence it is much more restrictive. In [18] we considered unitary symmetry, i.e. $G=$ $U(d)$ and $\mathcal{H}_{A}=\mathcal{H}_{B}=\mathbb{C}^{d}$, whereas in [19] we analyzed orthogonal symmetry with $G=O(d) \subset U(d)$. It turns out that contrary to the symmetric states considered in $[16,17]$ the states constructed in $[18,19]$ give rise to simple separability criteria. In the present paper we construct multipartite states with rotational $\mathrm{SO}(3)$ symmetry.

The paper is organized as follows: in Section 2 we recall basic properties of rotationally invariant bipartite states. This section summarizes the main results obtained in $[5,6,7,8]$. In section 3 we construct multipartite $\mathrm{SO}(3)$-invariant states and study its basic properties: separability and multiPPT conditions. More technical analysis is moved to appendixes. Final conclusions are collected in the last section.

\section{Rotationally invariant bipartite states}

\subsection{Werner-like states}

Let us consider two particles with spins $j_{A}$ and $j_{B} \geq j_{A}$. The composed bipartite system lives in $\mathcal{H}_{A B}=\mathcal{H}_{A} \otimes \mathcal{H}_{B}$, with $\mathcal{H}_{A}=\mathbb{C}^{d_{A}}$ and $\mathcal{H}_{B}=\mathbb{C}^{d_{B}}$, where $d_{A}=2 j_{A}+1$ and $d_{B}=2 j_{B}+1$. Recall that the Hilbert space corresponding to spin- $j$ particle is spanned by $d=2 j+1$ eigenstates $|j, m\rangle$, where 
$m=-j,-j+1, \ldots, j$. A bipartite operator $\rho$ is said to be Werner-like rotationally or $\mathrm{SO}(3)$-invariant iff for any $R \in \mathrm{SO}(3)$

$$
\left[\mathfrak{D}^{\left(j_{A}\right)}(R) \otimes \mathfrak{D}^{\left(j_{B}\right)}(R), \rho\right]=0,
$$

where $\mathfrak{D}^{(j)}(R)$ denotes irreducible unitary representation of $R$ in $\mathbb{C}^{2 j+1}$. As is well known the tensor product of two irreducible representations $\mathfrak{D}^{\left(j_{A}\right)}(R) \otimes \mathfrak{D}^{\left(j_{B}\right)}(R)$ is no longer irreducible in $\mathbb{C}^{d_{A}} \otimes \mathbb{C}^{d_{B}}$. It decomposes into a direct sum of irreducible representations

$$
\mathfrak{D}^{\left(j_{A}\right)}(R) \otimes \mathfrak{D}^{\left(j_{B}\right)}(R)=\bigoplus_{J=j_{B}-j_{A}}^{j_{B}+j_{A}} \mathfrak{D}^{(J)}(R)
$$

each appearing with multiplicity 1 . The composite space $\mathcal{H}_{A B}$ is spanned by $d_{A} \cdot d_{B}$ vectors $|J M\rangle$ with $J=j_{B}-j_{A}, \ldots, j_{B}+j_{A}$ and $M=-J, \ldots, J$, that is

$$
|J M\rangle=\sum_{m_{A}, m_{B}}\left\langle j_{A}, m_{A} ; j_{B}, m_{B} \mid J M\right\rangle\left|m_{A} ; m_{B}\right\rangle
$$

where $\left\langle j_{A}, m_{A} ; j_{B}, m_{B} \mid J M\right\rangle$ denote Clebsh-Gordan coefficients [20, 21, 22], and

$$
\left|m_{A} ; m_{B}\right\rangle=\left|j_{A}, m_{A}\right\rangle \otimes\left|j_{B}, m_{B}\right\rangle .
$$

Now, the space of Werner-like $\mathrm{SO}(3)$-invariant operator is spanned by $2 j_{A}+1$ projectors:

$$
Q^{J}=\sum_{M=-J}^{J}|J M\rangle\langle J M|
$$

that is, any $\mathrm{SO}(3)$-invariant operator may be written as follows

$$
\rho=\sum_{J} q_{J} \widetilde{Q}^{J}
$$

where $q_{J} \geq 0$ with $\sum_{J} q_{J}=1$, and we use the following notation $\widetilde{A}=A / \operatorname{Tr} A$. Note that $\operatorname{Tr} Q^{J}=$ $2 J+1$.

It is evident that an arbitrary bipartite state $\rho$ may be projected onto the $\mathrm{SO}(3)$-invariant subspace by the following twirl operation:

$$
\mathbb{T}(\rho)=\int \mathfrak{D}^{\left(j_{A} \otimes j_{B}\right)}(R) \rho\left[\mathfrak{D}^{\left(j_{A} \otimes j_{B}\right)}(R)\right]^{\dagger} d R
$$

where $d R$ is an invariant normalized Haar measure on $\mathrm{SO}(3)$, and we introduce the following slightly more compact notation:

$$
\mathfrak{D}^{\left(j_{A} \otimes j_{B}\right)}(R)=\mathfrak{D}^{\left(j_{A}\right)}(R) \otimes \mathfrak{D}^{\left(j_{B}\right)}(R) .
$$

Clearly, $\mathbb{T}(\rho)$ is of the form (2.6) with fidelities $q_{J}=\operatorname{Tr}\left(\rho Q^{J}\right)$. 


\subsection{Isotropic-like states}

Now, a bipartite state $\rho$ is isotropic-like $\mathrm{SO}(3)$-invariant iff

$$
\left[\mathfrak{D}^{\left(j_{A}\right)}(R) \otimes \overline{\mathfrak{D}^{\left(j_{B}\right)}(R)}, \rho\right]=0
$$

where $\overline{\mathfrak{D}^{(j)}(R)}$ denotes conjugate representation. Representations $\mathfrak{D}^{(j)}$ and $\overline{\mathfrak{D}^{(j)}}$ are equivalent and hence there exists an intertwining unitary operator $V$ such that $V \mathfrak{D}^{(j)}=\overline{\mathfrak{D}^{(j)}} V$. It turns out that

$$
V|j, m\rangle=(-1)^{j-m}|j,-m\rangle .
$$

Let us define a family of projectors

$$
P^{J}=(\mathbb{1} \otimes V) Q^{J}\left(\mathbb{1} \otimes V^{\dagger}\right) .
$$

Note, that $P^{J}$ are $\mathfrak{D}^{\left(j_{A} \otimes \overline{j_{B}}\right)}$-invariant, where in analogy to (2.8), we introduced

$$
\mathfrak{D}^{\left(j_{A} \otimes \overline{j_{B}}\right)}(R)=\mathfrak{D}^{\left(j_{A}\right)}(R) \otimes \overline{\mathfrak{D}^{\left(j_{B}\right)}(R)} .
$$

Indeed, one has

$$
\begin{aligned}
\mathfrak{D}^{\left(j_{A} \otimes \overline{j_{B}}\right.}(R) P^{J} & =\mathfrak{D}^{\left(j_{A} \otimes \overline{j_{B}}\right)}(R)(\mathbb{1} \otimes V) Q^{J}\left(\mathbb{1} \otimes V^{\dagger}\right)=(\mathbb{1} \otimes V) \mathfrak{D}^{\left(j_{A} \otimes j_{B}\right)}(R) Q^{J}\left(\mathbb{1} \otimes V^{\dagger}\right) \\
& =(\mathbb{1} \otimes V) Q^{J} \mathfrak{D}^{\left(j_{A} \otimes j_{B}\right)}(R)\left(\mathbb{1} \otimes V^{\dagger}\right)=(\mathbb{1} \otimes V) Q^{J}\left(\mathbb{1} \otimes V^{\dagger}\right) \mathfrak{D}^{\left(j_{A} \otimes \overline{j_{B}}\right.}(R) \\
& =P^{J} \mathfrak{D}^{\left(j_{A} \otimes \overline{j_{B}}\right.}(R) .
\end{aligned}
$$

Therefore, any $\mathfrak{D}^{\left(j_{A} \otimes \overline{j_{B}}\right)}$-invariant state has the following form

$$
\rho=\sum_{J} p_{J} \widetilde{P}^{J}
$$

where $p_{J} \geq 0$ with $\sum_{J} p_{J}=1$. Again, an arbitrary bipartite state $\rho$ may be projected onto the

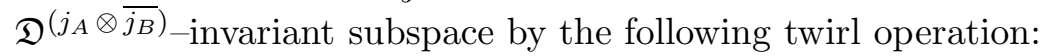

$$
\mathbb{T}^{\prime}(\rho)=\int \mathfrak{D}^{\left(j_{A} \otimes \overline{j_{B}}\right)}(R) \rho\left[\mathfrak{D}^{\left(j_{A} \otimes \overline{j_{B}}\right)}(R)\right]^{\dagger} d R
$$

where $d R$ is an invariant normalized Haar measure on $\mathrm{SO}(3)$. Clearly, $\mathbb{T}^{\prime}(\rho)$ is of the form (2.13) with fidelities $p_{J}=\operatorname{Tr}\left(\rho P_{J}\right)$.

\section{$2.3 \quad$ PPT states}

Note, that both families of $\mathrm{SO}(3)$-invariant states, i.e. Werner-like states (2.6) and isotropic-like states (2.13) are not independent. They are related by a partial transposition $\mathbb{1} \otimes \tau$, i.e. $\rho$ is $\mathfrak{D}^{j_{A}} \otimes j_{B_{-}}$ invariant (it belongs to the class $(2.6))$ iff $(\mathbb{1} \otimes \tau) \rho$ is $\mathfrak{D}^{j_{A}} \otimes \overline{j_{B}}$-invariant. Equivalently, using twirl operations $\mathbb{T}$ and $\mathbb{T}^{\prime}$ one has

$$
\mathbb{T}^{\prime}=(\mathbb{1} \otimes \tau) \circ \mathbb{T} \circ(\mathbb{1} \otimes \tau)
$$




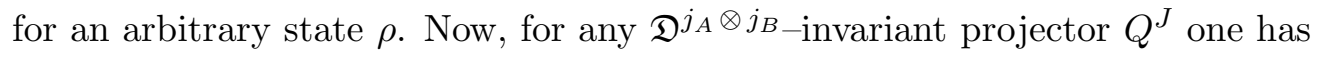

$$
(\mathbb{1} \otimes \tau) \widetilde{Q}^{J}=\sum_{J^{\prime}} X_{J J^{\prime}} \widetilde{P}_{J^{\prime}},
$$

where the $d_{A} \times d_{A}$ matrix $\mathbf{X}=\left[\mathbf{X}_{J J^{\prime}}\right]$ reads as follows

$$
X_{J J^{\prime}}=\operatorname{Tr}\left[(\mathbb{1} \otimes \tau) \widetilde{Q}^{J} P^{J^{\prime}}\right] .
$$

Note that due to $\sum_{J} P^{J}=I_{d_{A}} \otimes I_{d_{B}}$ one finds

$$
\sum_{J^{\prime}} X_{J J^{\prime}}=1
$$

However the matrix elements $X_{J J^{\prime}}$ are not necessarily positive which prevents $X$ to be a stochastic matrix. Interestingly, matrix $X$ satisfies

$$
X^{2}=I
$$

where I stands for $d_{A} \times d_{A}$ identity matrix which implies $X^{-1}=X$ (for proof see Appendix A).

It turns out that using several properties of Clebsch-Gordan coefficients matrix $X_{J J^{\prime}}$ may be expressed in terms of so called 6- $j$ Wigner symbol well known from the quantum theory of angular momentum [20]. Following [7] we show in the Appendix B that $X_{J J^{\prime}}$ may be expressed as follows:

$$
X_{J J^{\prime}}=(-1)^{2 j_{B}}\left(2 J^{\prime}+1\right)\left\{\begin{array}{lll}
j_{A} & j_{B} & J \\
j_{A} & j_{B} & J^{\prime}
\end{array}\right\},
$$

where the curly brackets denote a 6- $j$ Wigner symbol [20]. Equivalently, using the Racah $W$-coefficients

$$
W\left(j_{A}, j_{B}, j_{A}^{\prime}, j_{B}^{\prime} ; J J^{\prime}\right)=(-1)^{\alpha}\left\{\begin{array}{ccc}
j_{A} & j_{B} & J \\
j_{A}^{\prime} & j_{B}^{\prime} & J^{\prime}
\end{array}\right\}
$$

where $\alpha=j_{A}+j_{B}+j_{A}^{\prime}+j_{B}^{\prime}$, one finds

$$
X_{J J^{\prime}}=(-1)^{2 j_{A}}\left(2 J^{\prime}+1\right) W\left(j_{A}, j_{B}, j_{A}, j_{B} ; J J^{\prime}\right) .
$$

Therefore, if $\rho$ is given by (2.6), then its partial transposition has the following form:

$$
(\mathbb{1} \otimes \tau) \rho=\sum_{J} q_{J}^{\prime} \widetilde{P}^{J}
$$

where

$$
q_{J}^{\prime}=\sum_{J^{\prime}} q_{J^{\prime}} \mathbf{X}_{J^{\prime} J} .
$$

An $\mathrm{SO}(3)$-invariant state (2.6) is PPT iff $q_{J}^{\prime} \geq 0$ for all $J=j_{B}-j_{A}, \ldots, j_{B}+j_{A}$.

Conversely, if $\rho$ is given by (2.13), then its partial transposition has the following form:

$$
(\mathbb{1} \otimes \tau) \rho=\sum_{J} p_{J}^{\prime} \widetilde{Q}^{J},
$$


with

$$
p_{J}^{\prime}=\sum_{J^{\prime}} p_{J^{\prime}} X_{J^{\prime} J}
$$

where we used the fact that $X^{-1}=X$. An $\mathrm{SO}(3)$-invariant state $(2.13)$ is PPT iff $p_{J}^{\prime} \geq 0$ for all $J=j_{B}-j_{A}, \ldots, j_{B}+j_{A}$.

In Appendix $\mathrm{C}$ we show that for $j_{B} \geq j_{A}=1 / 2$ the $2 \times 2$ matrix $\mathbf{X}$ reads as follows:

$$
X=\frac{1}{2 j_{B}+1}\left(\begin{array}{cc}
-1 & 2\left(j_{B}+1\right) \\
2 j_{B} & 1
\end{array}\right) \text {. }
$$

For $j_{B} \geq j_{A}=1$ the corresponding $3 \times 3$ matrix $X$ is given by (Appendix $\mathrm{C}$ ):

$$
X=\frac{1}{j_{B}\left(j_{B}+1\right)\left(2 j_{B}+1\right)}\left(\begin{array}{ccc}
j_{B}+1 & -\left(j_{B}+1\right)\left(2 j_{B}+1\right) & j_{B}\left(j_{B}+1\right)\left(2 j_{B}+3\right) \\
-\left(j_{B}+1\right)\left(2 j_{B}-1\right) & \left(j_{B}^{2}+j_{B}-1\right)\left(2 j_{B}+1\right) & j_{B}\left(2 j_{B}+3\right) \\
j_{B}\left(j_{B}+1\right)\left(2 j_{B}-1\right) & j_{B}\left(2 j_{B}+1\right) & j_{B}
\end{array}\right) .
$$

\subsection{Separability}

A Werner-like rotationally invariant state $\rho$ is separable iff there exists a separable state $\sigma$ in $\mathcal{H}_{A B}$ such that

$$
\rho=\mathbb{T}(\sigma) .
$$

Moreover, it is clear that pure separable states $\varphi \otimes \psi \in \mathcal{H}_{A B}$ are mapped via twirl into the extremal separable symmetric states $\mathbb{T}(|\varphi \otimes \psi\rangle\langle\varphi \otimes \psi|)$. Note that among invariant projectors $Q^{J}$ only one with maximal $J=j_{A}+j_{B}$ is separable since

$$
\widetilde{Q}^{j_{A}+j_{B}}=\mathbb{T}\left(\left|j_{A} ; j_{B}\right\rangle\left\langle j_{A} ; j_{B}\right|\right)
$$

If $J \neq j_{A}+j_{B}$ the corresponding $Q^{J}$ is not PPT and hence it is not separable. It is well known $[5,6,7,8]$ that for $j_{A}=1 / 2$ and arbitrary $j_{B}$ rotationally invariant state is separable iff it is PPT, i.e.

$$
\rho=q_{j_{B}-1 / 2} \widetilde{Q}^{j_{B}-1 / 2}+q_{j_{B}+1 / 2} \widetilde{Q}^{j_{B}-1 / 2},
$$

with $q_{j_{B}-1 / 2}, q_{j_{B}+1 / 2} \geq 0$ and $q_{j_{B}-1 / 2}+q_{j_{B}+1 / 2}=1$, is separable iff

$$
q_{J}^{\prime}=\sum_{J^{\prime}=j_{B}-1 / 2}^{j_{B}+1 / 2} q_{J^{\prime}} \mathbf{X}_{J^{\prime} J} \geq 0,
$$

with $\mathbf{X}$ given in (2.26). It gives therefore the following necessary and sufficient condition for separability

$$
q_{j_{B}+1 / 2} \geq \frac{1}{d_{B}}
$$


Note that PPT states define a convex set - an interval $\left[\mathbf{q}, \mathbf{q}^{\prime}\right]$, with $\mathbf{q}=(0,1)$ and $\mathbf{q}^{\prime}=\left(\left(d_{B}-\right.\right.$ $\left.1) / d_{B}, 1 / d_{B}\right)$, where $\mathbf{q}=\left(q_{j_{B}-1 / 2}, q_{j_{B}+1 / 2}\right)$. Clearly, a state corresponding to $\mathbf{q}$ is separable - it is $\widetilde{Q}^{j_{B}+1 / 2}$. To show that a state corresponding to $\mathbf{q}^{\prime}$ is also separable let us observe that

$$
\operatorname{Tr}\left(\sigma Q^{j_{B}+1 / 2}\right)=\frac{1}{d_{B}}, \quad \operatorname{Tr}\left(\sigma Q^{j_{B}-1 / 2}\right)=\frac{d_{B}-1}{d_{B}},
$$

where e.g. $\sigma=\left|-1 / 2 ; j_{B}\right\rangle\left\langle-1 / 2 ; j_{B}\right|$. The same result holds for $\sigma=\left|1 / 2 ;-j_{B}\right\rangle\left\langle 1 / 2 ;-j_{B}\right|$.

Similarly, an isotropic-like rotationally invariant state in $\mathbb{C}^{2} \otimes \mathbb{C}^{d_{B}}$ is separable iff it is PPT, that is

$$
\rho=p_{j_{B}-1 / 2} \widetilde{P}^{j_{B}-1 / 2}+p_{j_{B}+1 / 2} \widetilde{P}^{j_{B}+1 / 2},
$$

with $p_{j_{B}-1 / 2}, p_{j_{B}+1 / 2} \geq 0$ and $p_{j_{B}-1 / 2}+p_{j_{B}+1 / 2}=1$, is separable iff

$$
p_{j_{B}+1 / 2} \geq \frac{1}{d_{B}} .
$$

Another interesting case is when $j_{B} \geq j_{A}=1$. It was shown $[7,8]$ that for integer $j_{B}$, i.e. odd $d_{B}=2 j_{B}+1$, rotationally invariant state is separable iff it is PPT. However, for half-integer $j_{B}$ (even $\left.d_{B}\right)$ there exist bound entangled states, i.e. PPT but entangled. Now, using (2.27), for integer $j_{B}$ a rotationally invariant state

$$
\rho=q_{j_{B}-1} \widetilde{Q}^{j_{B}-1}+q_{j_{B}} \widetilde{Q}^{j_{B}}+q_{j_{B}+1} \widetilde{Q}^{j_{B}+1}
$$

is separable iff

$$
\begin{aligned}
q_{j_{B}-1} d_{B}-q_{j_{B}}\left(j_{B}^{2}-1\right) & \leq 1 \\
q_{j_{B}}\left(2 j_{B}^{2}+j_{B}-1\right)-q_{j_{B}-1}\left(1-2 j_{B}^{2}+j_{B}\right) & \leq j_{B} d_{B} .
\end{aligned}
$$

The above conditions considerably simplify for $j_{B}=1$. One obtains

$$
q_{0} \leq \frac{1}{3}, \quad q_{1} \leq \frac{1}{2}
$$

which reproduce separability conditions for $O(3) \otimes O(3)$-invariant states (see formula (27) in [19]). Similar results hold for isotropic-like rotationally invariant states with $j_{B} \geq j_{A}=1$. For $j_{B} \geq j_{A}>1$ the situation is much more complicated. For some partial results consult $[5,6,7,8]$.

\subsection{Special case: $j_{A}=j_{B}$}

Consider now the special case when both particles have the same spin $j_{A}=j_{B} \equiv j$. One has two families of projectors:

$$
Q^{0}, Q^{1}, \ldots, Q^{d-1}
$$

and

$$
P^{0} \equiv P_{d}^{+}, P^{1}, \ldots, P^{d-1}
$$

where $d=2 j+1$,

$$
P_{d}^{+}=\frac{1}{d} \sum_{m_{A}, m_{B}=-j}^{j}\left|m_{A} ; m_{B}\right\rangle\left\langle m_{A} ; m_{B}\right|,
$$

denotes a projector onto the maximally entangled state. Using definitions (2.5) and (2.11) and properties of the Clebsch-Gordan coefficients one proves the following 
Theorem 1 The Schmidt number [23] of $Q^{J}$ and $P^{J}$ is given by

$$
\mathrm{SN}\left(Q^{J}\right)=\mathrm{SN}\left(P^{J}\right)=d-J,
$$

for $J=0,1, \ldots, d-1$.

Note, that in the case of the standard $U \otimes U$-invariant Werner state one has only two projectors: $Q^{d-2}$ and $Q^{d-1}$. $Q^{d-2}$ has Schmidt number 2 and $Q^{d-1}$ is separable. Therefore, contrary to the 1-parameter family of Werner states the $(d-1)$-parameter family of Werner-like $\mathrm{SO}(3)$-invariant states gives rise to the full spectrum of entangled states: from separable one to states with the maximal Schmidt number $d$. In the case of isotropic $U \otimes \bar{U}$-invariant state one has maximally entangled (i.e. with Schmidt number $d$ ) $P^{0}=P_{d}^{+}$and separable $P^{d-1}$.

The matrix $X_{J J^{\prime}}$ given by (2.38) simplifies to

$$
X_{J J^{\prime}}=(-1)^{d-1}\left(2 J^{\prime}+1\right)\left\{\begin{array}{lll}
j & j & J \\
j & j & J^{\prime}
\end{array}\right\},
$$

In particular for $j=1 / 2$ the formula (2.26) reconstructs $X$ matrix for the Werner $U \otimes U$-invariant states in $\mathbb{C}^{2} \otimes \mathbb{C}^{2}$ (see formula (15) in $[18]$ ):

$$
X=\frac{1}{2}\left(\begin{array}{rr}
-1 & 3 \\
1 & 1
\end{array}\right) \text {. }
$$

For $j=1$ the formula (2.27) reconstructs $X$ matrix for the orthogonally $O(3) \otimes O(3)$-invariant states in $\mathbb{C}^{3} \otimes \mathbb{C}^{3}$ (see formula (30) in $[19]$ ):

$$
X=\frac{1}{6}\left(\begin{array}{rrc}
2 & -6 & 10 \\
-2 & 3 & 5 \\
2 & 3 & 1
\end{array}\right)
$$

\section{Multipartite $\mathrm{SO}(3)$ symmetric states}

\subsection{Werner-like family}

Consider now $2 K$-partite system living in $\mathcal{H}_{A} \otimes \mathcal{H}_{B}$, where

$$
\mathcal{H}_{A}=\mathcal{H}_{1} \otimes \ldots \otimes \mathcal{H}_{K}
$$

and

$$
\mathcal{H}_{B}=\mathcal{H}_{K+1} \otimes \ldots \otimes \mathcal{H}_{2 K}
$$

with $\mathcal{H}_{1}=\ldots=\mathcal{H}_{K}=\mathbb{C}^{d_{A}}$ and $\mathcal{H}_{K+1}=\ldots=\mathcal{H}_{2 K}=\mathbb{C}^{d_{B}}$. Let $\mathbf{R}=\left(R_{1}, \ldots, R_{K}\right)$ with $R_{i} \in S O(3)$ and define

$$
\mathfrak{D}^{\left(j_{A}\right)}(\mathbf{R}) \otimes \mathfrak{D}^{\left(j_{B}\right)}(\mathbf{R})=\bigotimes_{i=1}^{K} \mathfrak{D}^{\left(j_{A} \otimes j_{B}\right)}\left(R_{i}\right),
$$

where for each $i=1, \ldots, K$ a bipartite unitary operator $\mathfrak{D}^{\left(j_{A} \otimes j_{B}\right)}\left(R_{i}\right)$ acts on $\mathcal{H}_{i} \otimes \mathcal{H}_{K+i}$. Now, we call a $2 K$-partite state a Werner-like $\mathrm{SO}(3)$-invariant iff

$$
\left[\mathfrak{D}^{\left(j_{A}\right)}(\mathbf{R}) \otimes \mathfrak{D}^{\left(j_{B}\right)}(\mathbf{R}), \rho\right]=0,
$$


for any $\mathbf{R} \in S O(3) \times \ldots \times S O(3)$. To parameterize the set of $2 K$-partite invariant states let us introduce the following set of projectors:

$$
\mathbf{Q}^{\mathbf{J}}=Q_{1 \mid K+1}^{J_{1}} \otimes \ldots \otimes Q_{K \mid 2 K}^{J_{K}}
$$

where $\mathbf{J}=\left(J_{1}, \ldots, J_{k}\right)$ is a $K$-vector with $J_{i}=j_{B}-j_{A}, \ldots, j_{B}+j_{A}$. It is clear that

1. $\mathbf{Q}^{\mathbf{J}}$ are $\mathrm{SO}(3)$-invariant,

2. $\mathbf{Q}^{\mathbf{J}} \cdot \mathbf{Q}^{\mathbf{J}^{\prime}}=\delta_{\mathbf{J} \mathbf{J}^{\prime}} \mathbf{Q}^{\mathbf{J}}$,

3. $\sum_{\mathbf{J}} \mathbf{Q}^{\mathbf{J}}=\left(I_{d_{A}} \otimes I_{d_{B}}\right) \otimes K$.

Therefore, an arbitrary $2 K$-partite $\mathrm{SO}(3)$-invariant state has the following form

$$
\rho=\sum_{\mathbf{J}} q_{\mathbf{J}} \widetilde{\mathbf{Q}}^{\mathbf{J}}
$$

with $q_{\mathbf{J}} \geq 0$ and $\sum_{\mathbf{J}} q_{\mathbf{J}}=1$. Hence, the set of rotationally invariant states defines $\left(d_{A}^{K}-1\right)$-dimensional simplex.

\subsection{Isotropic-like family}

It is clear that we may use the same scheme to define $2 k$-partite isotropic-like states. For any $\mathbf{R}=$ $\left(R_{1}, \ldots, R_{K}\right)$ with $R_{i} \in S O(3)$ one defines

$$
\mathfrak{D}^{\left(j_{A}\right)}(\mathbf{R}) \otimes \overline{\mathfrak{D}^{\left(j_{B}\right)}(\mathbf{R})}=\bigotimes_{i=1}^{K} \mathfrak{D}^{\left(j_{A} \otimes \overline{j_{B}}\right)}\left(R_{i}\right),
$$

where for each $i=1, \ldots, K$ a bipartite unitary operator $\left.\mathfrak{D}^{\left(j_{A} \otimes \overline{j_{B}}\right.}\right)\left(R_{i}\right)$ acts on $\mathcal{H}_{i} \otimes \mathcal{H}_{K+i}$. Now, we call a $2 K$-partite state an isotropic-like $\mathrm{SO}(3)$-invariant iff

$$
\left[\mathfrak{D}^{\left(j_{A}\right)}(\mathbf{R}) \otimes \overline{\mathfrak{D}^{\left(j_{B}\right)}(\mathbf{R})}, \rho\right]=0,
$$

for any $\mathbf{R} \in S O(3) \times \ldots \times S O(3)$. To parameterize the set of $2 K$-partite invariant states let us introduce the following set of projectors:

$$
\mathbf{P}^{\mathbf{J}}=P_{1 \mid K+1}^{J_{1}} \otimes \ldots \otimes P_{K \mid 2 K}^{J_{K}},
$$

where $\mathbf{J}=\left(J_{1}, \ldots, J_{k}\right)$ is a $K$-vector with $J_{i}=j_{B}-j_{A}, \ldots, j_{B}+j_{A}$. It is clear that

1. $\mathbf{P}^{\mathbf{J}}$ are $\mathrm{SO}(3)$-invariant,

2. $\mathbf{P}^{\mathbf{J}} \cdot \mathbf{P}^{\mathbf{J}^{\prime}}=\delta_{\mathbf{J} \mathbf{J}^{\prime}} \mathbf{P}^{\mathbf{J}}$,

3. $\sum_{\mathbf{J}} \mathbf{P}^{\mathbf{J}}=\left(I_{d_{A}} \otimes I_{d_{B}}\right)^{\otimes K}$.

Therefore, an arbitrary $2 K$-partite $\mathrm{SO}(3)$-invariant state has the following form

$$
\rho=\sum_{\mathbf{J}} p_{\mathbf{J}} \widetilde{\mathbf{P}}^{\mathbf{J}}
$$

with $p_{\mathbf{J}} \geq 0$ and $\sum_{\mathbf{J}} p_{\mathbf{J}}=1$. The set of rotationally invariant states defines $\left(d_{A}^{K}-1\right)$-dimensional simplex. 


\section{$3.3 \sigma$-PPT states}

Now, following [18] let us introduce the family of partial transpositions parameterized by a binary $K$-vector $\boldsymbol{\sigma}=\left(\sigma_{1}, \ldots, \sigma_{K}\right)$ :

$$
\tau_{\boldsymbol{\sigma}}=\mathbb{1}^{\otimes K} \otimes \tau^{\sigma_{1}} \otimes \ldots \otimes \tau^{\sigma_{K}}
$$

where $\tau^{\alpha}=\mathbb{1}$ for $\alpha=0$ and $\tau^{\alpha}=\tau$ for $\alpha=1$. A $2 K$-partite state $\rho$ is $\boldsymbol{\sigma}$-PPT iff $\tau_{\boldsymbol{\sigma}} \rho \geq 0$. In terms of coefficients $q_{\mathbf{J}}$ the property of $\boldsymbol{\sigma}$-PPT leads to the following conditions

$$
\sum_{\mathbf{J}} q_{\mathbf{J}} \mathbf{X}_{\mathbf{J J}^{\prime}}^{\boldsymbol{\sigma}} \geq 0
$$

for all $\mathbf{J}^{\prime}$. The $d_{A}^{K} \times d_{A}^{K}$ matrix $\mathbf{X}_{\mathbf{J} \mathbf{J}^{\prime}}^{\boldsymbol{\sigma}}$ is given by

$$
\mathbf{X}_{\mathbf{J} \mathbf{J}^{\prime}}^{\boldsymbol{\sigma}}=\operatorname{Tr}\left[\left(\tau_{\boldsymbol{\sigma}} \widetilde{\mathbf{Q}}^{\mathbf{J}}\right) \cdot \mathbf{P}_{\mathbf{J}^{\prime}}\right]
$$

Let us observe that

$$
\mathbf{X}^{\sigma}=X^{\sigma_{1}} \otimes \ldots \otimes X^{\sigma_{K}}
$$

where $X$ is defined by in (2.21). In component notation one finds

$$
\mathbf{X}_{\mathbf{J J}^{\prime}}^{\boldsymbol{\sigma}}=X_{J_{1} J_{1}^{\prime}}^{\sigma_{1}} \ldots X_{J_{K} J_{K}^{\prime}}^{\sigma_{K}}
$$

Again, in analogy to (2.18) and (2.19) one has

$$
\sum_{J^{\prime}} \mathbf{X}_{\mathbf{J J}^{\prime}}^{\boldsymbol{\sigma}}=1
$$

and $\mathbf{X}^{\boldsymbol{\sigma}} \cdot \mathbf{X}^{\boldsymbol{\sigma}}=I^{\otimes K}$ for any $\boldsymbol{\sigma}$. Therefore

$$
\left(\mathbf{X}^{\boldsymbol{\sigma}}\right)^{-1}=\mathbf{X}^{\boldsymbol{\sigma}}
$$

In the same way one defines a $\boldsymbol{\sigma}$-PPT subset of isotropic-like $2 K$-partite symmetric states. A state $\rho$ from the family (3.21) is $\boldsymbol{\sigma}$-PPT iff $\tau_{\boldsymbol{\sigma}} \rho \geq 0$, that is

$$
\sum_{\mathbf{J}} p_{\mathbf{J}} \mathbf{X}_{\mathbf{J J}^{\prime}}^{\boldsymbol{\sigma}} \geq 0
$$

for all $\mathbf{J}^{\prime}$.

\section{$3.4 \sigma$-invariance}

Note, that each binary vector $\boldsymbol{\sigma}$ gives rise to the new $2 K$-partite family of symmetric states. We call a state $\rho \boldsymbol{\sigma}$-invariant iff $\tau_{\boldsymbol{\sigma}} \rho$ is Werner-like invariant. To parameterize this family let us introduce the following set of bipartite operators:

$$
\Pi_{(\sigma)}^{J}=\left\{\begin{array}{ll}
Q^{J}, & \sigma=0 \\
P^{J}, & \sigma=1
\end{array} .\right.
$$

This operators may be used to construct a set of $2 K$-partite projectors

$$
\Pi_{(\boldsymbol{\sigma})}^{\mathbf{J}}=\Pi_{\left(\sigma_{1}\right) 1 \mid K+1}^{J_{1}} \otimes \ldots \otimes \Pi_{\left(\sigma_{K}\right) K \mid 2 K}^{J_{K}},
$$

satisfying 
1. $\boldsymbol{\Pi}_{(\boldsymbol{\sigma})}^{\mathbf{J}}$ are $\boldsymbol{\sigma}$-invariant,

2. $\boldsymbol{\Pi}^{\mathbf{J}}(\boldsymbol{\sigma}) \cdot \boldsymbol{\Pi}_{(\boldsymbol{\sigma})}^{\mathbf{J}^{\prime}}=\delta_{\mathbf{J} \mathbf{J}^{\prime}} \boldsymbol{\Pi}_{(\boldsymbol{\sigma})}^{\mathbf{J}}$,

3. $\sum_{\mathbf{J}} \boldsymbol{\Pi}_{(\boldsymbol{\sigma})}^{\mathbf{J}}=\left(I_{d_{A}} \otimes I_{d_{B}}\right) \otimes K$.

Therefore, an arbitrary $2 K$-partite $\boldsymbol{\sigma}$-invariant state has the following form

$$
\rho=\sum_{\mathbf{J}} \pi_{\mathbf{J}} \widetilde{\boldsymbol{\Pi}}_{(\boldsymbol{\sigma})}^{\mathbf{J}}
$$

with $\pi_{\mathbf{J}} \geq 0$ and $\sum_{\mathbf{J}} \pi_{\mathbf{J}}=1$. Clearly, the set of $\boldsymbol{\sigma}$-invariant states defines $\left(d_{A}^{K}-1\right)$-dimensional simplex. Let us note that for any two binary vectors $\boldsymbol{\mu}$ and $\boldsymbol{\nu}$ if $\rho$ is $\boldsymbol{\mu}$-invariant then $\tau_{\boldsymbol{\nu}} \rho$ is $(\boldsymbol{\mu} \oplus \boldsymbol{\nu})$-invariant, where $\boldsymbol{\mu} \oplus \boldsymbol{\nu}$ denotes addition $\bmod 2$.

\subsection{Separability}

A $2 K$-partite Werner-like rotationally invariant state $\rho$ is separable iff there exists a separable state $\sigma$ in $\mathcal{H}_{\text {total }}$ such that

$$
\rho=\mathbb{T}_{K}(\sigma)
$$

where $\mathbb{T}_{K}$ denotes $2 K$-partite twirl operation:

$$
\mathbb{T}_{K}(\omega)=\int\left[\mathfrak{D}^{\left(j_{A}\right)}(\mathbf{R}) \otimes \mathfrak{D}^{\left(j_{B}\right)}(\mathbf{R})\right] \omega\left[\mathfrak{D}^{\left(j_{A}\right)}(\mathbf{R}) \otimes \mathfrak{D}^{\left(j_{B}\right)}(\mathbf{R})\right]^{\dagger} d R_{1} \ldots d R_{K} .
$$

Moreover, it is clear that pure separable states $\varphi_{1} \otimes \ldots \otimes \varphi_{K} \otimes \psi_{1} \ldots \otimes \psi_{K} \in \mathcal{H}_{\text {total }}$ are mapped via twirl $\mathbb{T}_{K}$ into the extremal separable symmetric states. Again only one invariant projector $\mathbf{Q}^{\mathbf{J}}$ is separable - that corresponding to $\mathbf{J}=\left(j_{A}+j_{B}, \ldots, j_{A}+j_{B}\right)$. It is given by the twirl of $\left|j_{A}\right\rangle \otimes \ldots \otimes\left|j_{A}\right\rangle \otimes\left|j_{B}\right\rangle \otimes \ldots \otimes\left|j_{B}\right\rangle$.

Using techniques applied in $[18,19]$ one easily shows the following

Theorem 2 If $j_{B} \geq j_{A}=1 / 2$ or $j_{B} \geq j_{A}=1$ with integer $j_{B}$, then an arbitrary $\boldsymbol{\mu}$-invariant state $\rho$ is fully separable iff it is $\boldsymbol{\nu}$-PPT for all binary $K$-vectors $\boldsymbol{\nu}$. Moreover $\rho$ is $(1 \ldots K \mid K+1 \ldots 2 K)$ biseparable iff it is (1...1)-PPT.

In particular for $j_{A}=j_{B}=1 / 2$ the above theorem reconstructs separability conditions for $\boldsymbol{\mu}$ invariant states with unitary symmetry $U(2)$, see [18], whereas for $j_{A}=j_{B}=1$ one reconstructs separability conditions for $O(3)$-invariant states, see [19].

\subsection{Reductions}

It is evident that reducing the $2 K$ partite $\boldsymbol{\sigma}$-invariant state with respect to $\mathcal{H}_{i} \otimes \mathcal{H}_{i+K}$ pair one obtains $2(K-1)$-partite $\boldsymbol{\sigma}_{(i)}$-invariant state with

$$
\boldsymbol{\sigma}_{(i)}=\left(\sigma_{1}, \ldots, \check{\sigma}_{i}, \ldots, \sigma_{K}\right)
$$

where $\check{\sigma}_{i}$ denotes the omitting of $\sigma_{i}$. The reduced state lives in

$$
\mathcal{H}_{1} \otimes \ldots \check{\mathcal{H}}_{i} \otimes \ldots \otimes \check{\mathcal{H}}_{i+K} \otimes \ldots \otimes \mathcal{H}_{2 K}
$$


The corresponding fidelities of the reduced symmetric state are given by

$$
\pi_{\left(J_{1} \ldots J_{K}\right)}=\sum_{j=j_{B}-j_{A}}^{j_{B}+j_{A}} \pi_{\left(J_{1} \ldots J_{i-1} j J_{i+1} \ldots J_{K}\right)} .
$$

Note, that reduction with respect to a 'mixed' pair, say $\mathcal{H}_{i} \otimes \mathcal{H}_{j+K}$ with $j \neq i(i, j \leq K)$, is equivalent to two 'natural' reductions with respect to $\mathcal{H}_{i} \otimes \mathcal{H}_{i+K}$ and $\mathcal{H}_{j} \otimes \mathcal{H}_{j+K}$ and hence it gives rise to $2(K-$ $2)$-partite invariant state. This procedure establishes a natural hierarchy of multipartite invariant states.

\section{Conlusions}

We have introduced a new family of multipartite rotationally symmetric states for $2 K$ particles: $K$ spin- $j_{A}$ and $K$ spin- $j_{B}$ particles $\left(j_{B} \geq j_{A}\right)$. Within this class we have formulated separability conditions for $j_{B} \geq j_{A}=1 / 2$ and $j_{B} \geq j_{A}=1$ with integer $j_{B}$. It turned out that full $2 K$-separability is equivalent to multi $\boldsymbol{\sigma}$-PPT conditions with $\boldsymbol{\sigma}$ being a binary $K$-vector.

Recently, a detailed analysis of multipartite symmetric states and their application in quantum information theory was performed by Eggeling in his $\mathrm{PhD}$ thesis [17]. This construction may be applied for $\mathrm{SO}(3)$ symmetry as follows. Consider $N$ spin- $j$ particles. An $N$-partite state $\rho$ is rotationally invariant iff

$$
\left[\mathfrak{D}^{(j)}(R) \otimes \ldots \otimes \mathfrak{D}^{(j)}(R), \rho\right]=0
$$

for all $R \in S O(3)$. It is clear that the detailed parametrization of this class is highly nontrivial: it corresponds to addition of $N$ angular momenta and, as is well known even the case $N=3$ gives rise to considerable complications (see e.g. $[20,21,22]$ ). If $N=2 K$ our class defines only a commutative subclass within Eggeling's class.

Note, that our construction may be slightly generalized. Instead of $K$ spin- $j_{A}$ and $K \operatorname{spin}-j_{B}$ particles we may consider $2 K$ particles with arbitrary spins:

$$
\left(j_{A_{1}}, j_{B_{1}}\right),\left(j_{A_{2}}, j_{B_{2}}\right), \ldots,\left(j_{A_{K}}, j_{B_{K}}\right) .
$$

Now, a $2 K$-partite state $\rho$ is $\mathrm{SO}(3)$-invariant iff

$$
\left[\bigotimes_{i=1}^{K} \mathfrak{D}^{\left(j_{A_{i}}\right)}\left(R_{i}\right) \otimes \bigotimes_{i=1}^{K} \mathfrak{D}^{\left(j_{B_{i}}\right)}\left(R_{i}\right), \rho\right]=0,
$$

for all $R_{1}, \ldots, R_{K} \in S O(3)$. It is clear that such general situation does not apply for Eggeling's construction where all particles carry the same spins.

It is hoped that the multipartite state constructed in this paper may serve as a laboratory for testing various concepts from quantum information theory and they may shed new light on the more general investigation of multipartite entanglement. Note, that using duality between bipartite quantum states and quantum channels [24] one may consider rotationally invariant quantum channels transforming a state of $\operatorname{spin}-j_{B}$ particle into a state of spin- $j_{A}$ one. Relaxing positivity condition upon $\rho$ the above duality gives rise to rotationally invariant positive maps which may be used to detect quantum bipartite entanglement. In the multipartite case the situation is different. Now a crucial role is played 
by maps which are positive but only on separable states. Note that a tensor product of two positive maps is no longer positive but clearly it is positive on separable states. Therefore, our construction of multipartite symmetric states may be dually used to produce invariant classes of multi-linear maps which may serve as a useful tool in detecting multi-partite entanglement.

\section{Appendix A}

Using properties of the operator $V$ defined in (2.10) one shows that

$$
\operatorname{Tr}\left[(\mathbb{1} \otimes \tau) Q^{J} P^{J^{\prime}}\right]=\operatorname{Tr}\left[(\mathbb{1} \otimes \tau) Q^{J^{\prime}} P^{J}\right],
$$

that is,

$$
(2 J+1) X_{J J^{\prime}}=\left(2 J^{\prime}+1\right) X_{J^{\prime} J} .
$$

Now, following (2.17) one has

$$
\begin{aligned}
X_{J J^{\prime}}^{-1} & =\operatorname{Tr}\left[(\mathbb{1} \otimes \tau) \widetilde{P}^{J} Q^{J^{\prime}}\right] \\
& =\frac{1}{2 J+1} \operatorname{Tr}\left[(\mathbb{1} \otimes \tau) P^{J} Q^{J^{\prime}}\right],
\end{aligned}
$$

and using (A.1) one shows that $X_{J J^{\prime}}^{-1}=X_{J J^{\prime}}$, that is, $X^{2}=I$.

\section{Appendix B}

Using (2.5) and (2.11) one finds for the matrix $\mathbf{X}$ :

$$
\mathbf{X}_{J J^{\prime}}=\frac{1}{2 J+1} \sum_{M, M^{\prime}} \operatorname{Tr}\left[(\mathbb{1} \otimes \tau)|J M\rangle\left\langle J M|(\mathbb{1} \otimes V)| J^{\prime} M^{\prime}\right\rangle\left\langle J^{\prime} M^{\prime}\right|\left(\mathbb{1} \otimes V^{\dagger}\right)\right] .
$$

Therefore, taking into account (2.3) and the following relation between Clebsch-Gordan coefficients and $3-j$ Wigner symbols:

$$
\left\langle j_{1}, j_{2} ; m_{1}, m_{2} \mid J M\right\rangle=(-1)^{j_{1}-j_{2}+M} \sqrt{2 J+1}\left(\begin{array}{ccc}
j_{1} & j_{2} & J \\
m_{1} & m_{2} & -M
\end{array}\right),
$$

one obtains

$$
\begin{aligned}
& \operatorname{Tr}\left[(\mathbb{1} \otimes \tau)|J M\rangle\left\langle J M|(\mathbb{1} \otimes V)| J^{\prime} M^{\prime}\right\rangle\left\langle J^{\prime} M^{\prime}\right|\left(\mathbb{1} \otimes V^{\dagger}\right)\right] \\
& =(2 J+1)\left(2 J^{\prime}+1\right) \sum_{m_{A}, m_{B}} \sum_{l_{A}, l_{B}} \sum_{m_{A}^{\prime}, m_{B}^{\prime}} \sum_{l_{A}^{\prime}, l_{B}^{\prime}}(-1)^{2\left(M+M^{\prime}\right)} \delta_{m_{A}, l_{A}^{\prime}} \delta_{l_{A}, m_{A}^{\prime}} \delta_{m_{B},-m_{B}^{\prime}} \delta_{l_{B},-l_{B}^{\prime}} \\
& \times\left(\begin{array}{ccc}
j_{A} & j_{B} & J \\
m_{A} & m_{B} & -M
\end{array}\right)\left(\begin{array}{ccc}
j_{A} & j_{B} & J \\
l_{A} & l_{B} & -M
\end{array}\right)\left(\begin{array}{ccc}
j_{A} & j_{B} & J^{\prime} \\
m_{A}^{\prime} & m_{B}^{\prime} & -M^{\prime}
\end{array}\right)\left(\begin{array}{ccc}
j_{A} & j_{B} & J^{\prime} \\
l_{A}^{\prime} & l_{B}^{\prime} & -M^{\prime}
\end{array}\right) .
\end{aligned}
$$

Finally, using the symmetry of $3-j$ symbols

$$
\left(\begin{array}{ccc}
l_{1} & l_{2} & l_{3} \\
m_{1} & m_{2} & m_{3}
\end{array}\right)=(-1)^{l_{1}+l_{2}+l_{3}}\left(\begin{array}{ccc}
l_{1} & l_{2} & l_{3} \\
-m_{1} & -m_{2} & -m_{3}
\end{array}\right),
$$


and the following relation between $3-j$ and $6-j$ symbols

$$
\begin{aligned}
& (-1)^{l_{1}^{\prime}+l_{2}^{\prime}+l_{3}^{\prime}}\left\{\begin{array}{lll}
l_{1} & l_{2} & l_{3} \\
l_{1}^{\prime} & l_{2}^{\prime} & l_{3}^{\prime}
\end{array}\right\}=\sum_{m_{1}, m_{1}^{\prime}} \sum_{m_{2}, m_{2}^{\prime}} \sum_{m_{3}, m_{3}^{\prime}}(-1)^{m_{1}^{\prime}+m_{2}^{\prime}+m_{3}^{\prime}} \\
\times & \left(\begin{array}{ccc}
l_{1} & l_{2} & l_{3} \\
m_{1} & m_{2} & m_{3}
\end{array}\right)\left(\begin{array}{cccc}
l_{1} & l_{2}^{\prime} & l_{3}^{\prime} \\
-m_{1} & m_{2}^{\prime} & -m_{3}^{\prime}
\end{array}\right)\left(\begin{array}{ccc}
l_{1}^{\prime} & l_{2} & l_{3}^{\prime} \\
-m_{1}^{\prime} & -m_{2} & m_{3}^{\prime}
\end{array}\right)\left(\begin{array}{ccc}
l_{1}^{\prime} & l_{2}^{\prime} & l_{3} \\
m_{1}^{\prime} & -m_{2}^{\prime} & -m_{3}
\end{array}\right)
\end{aligned}
$$

one proves (2.38).

\section{Appendix $\mathrm{C}$}

The 6 - $j$-symbols are invariant under permutation of their columns, e.g.

$$
\left\{\begin{array}{lll}
j_{1} & j_{2} & j_{3} \\
J_{1} & J_{2} & J_{3}
\end{array}\right\}=\left\{\begin{array}{lll}
j_{2} & j_{1} & j_{3} \\
J_{2} & J_{1} & J_{3}
\end{array}\right\}
$$

and under exchange of two corresponding elements between rows, e.g.

$$
\left\{\begin{array}{lll}
j_{1} & j_{2} & j_{3} \\
J_{1} & J_{2} & J_{3}
\end{array}\right\}=\left\{\begin{array}{lll}
J_{1} & J_{2} & j_{3} \\
j_{1} & j_{2} & J_{3}
\end{array}\right\}
$$

Now, to find the $\mathbf{X}$ matrix for $j_{A}=1$ we shall use the following formulae [20]:

$$
\begin{gathered}
\left\{\begin{array}{ccc}
j_{1}-\frac{1}{2} & \frac{1}{2} & j_{1} \\
j_{2} & j & j_{2}-\frac{1}{2}
\end{array}\right\}=(-1)^{J}\left[\frac{(J+1)(J-2 j)}{2 j_{1}\left(2 j_{1}+1\right) 2 j_{2}\left(2 j_{2}+1\right)}\right]^{1 / 2}, \\
\left\{\begin{array}{ccc}
j_{1}-\frac{1}{2} & \frac{1}{2} & j_{1} \\
j_{2}-\frac{1}{2} & j & j_{2}
\end{array}\right\}=(-1)^{J-1 / 2}\left[\frac{\left(J-2 j_{1}+\frac{1}{2}\right)\left(J-2 j_{2}+\frac{1}{2}\right)}{2 j_{1}\left(2 j_{1}+1\right) 2 j_{2}\left(2 j_{2}+1\right)}\right]^{1 / 2},
\end{gathered}
$$

with $J=j_{1}+j_{2}+j$. Using these formulae together with symmetry relations (C.1)-(C.2) one obtains (2.26).

Now, to find the $\mathbf{X}$ matrix for $j_{A}=1$ we shall use the following formulae [20]:

$$
\begin{aligned}
\left\{\begin{array}{ccc}
j_{1}-1 & 1 & j_{1} \\
j_{2} & j & j_{2}-1
\end{array}\right\} & =(-1)^{J}\left[\frac{J(J+1)(J-2 j-1)(J-2 j)}{\left(2 j_{1}-1\right) 2 j_{1}\left(2 j_{1}+1\right)\left(2 j_{2}-1\right) 2 j_{2}\left(2 j_{2}+1\right)}\right]^{1 / 2}, \\
\left\{\begin{array}{ccc}
j_{1}-1 & 1 & j_{1} \\
j_{2}-1 & j & j_{2}
\end{array}\right\} & =(-1)^{J-1}\left[\frac{\left(J-2 j_{1}\right)(J-2 j+1)\left(J-2 j_{2}\right)\left(J-2 j_{2}+1\right)}{\left(2 j_{1}-1\right) 2 j_{1}\left(2 j_{1}+1\right)\left(2 j_{2}-1\right) 2 j_{2}\left(2 j_{2}+1\right)}\right]^{1 / 2}, \\
\left\{\begin{array}{ccc}
j_{1} & 1 & j_{1} \\
j_{2}-1 & j & j_{2}
\end{array}\right\} & =(-1)^{J}\left[\frac{2(J+1)(J-2 j)\left(J-2 j_{1}\right)\left(J-2 j_{2}+1\right)}{2 j_{1}\left(2 j_{1}+1\right)\left(2 j_{1}+2\right)\left(2 j_{2}-1\right) 2 j_{2}\left(2 j_{2}+1\right)}\right]^{1 / 2}, \\
\left\{\begin{array}{ccc}
j_{1} & 1 & j_{1} \\
j_{2} & j & j_{2}
\end{array}\right\} & =(-1)^{J} \frac{j(j+1)-j_{1}\left(j_{1}+1\right)-j_{2}\left(j_{2}+1\right)}{\left[j_{1}\left(2 j_{1}+1\right)\left(2 j_{1}+2\right) j_{2}\left(2 j_{2}+1\right)\left(2 j_{2}+2\right)\right]^{1 / 2}},
\end{aligned}
$$

together with symmetry relations (C.1)-(C.2). Simple calculations give (2.27). 


\section{Acknowledgments}

After this work was completed we have learnt from Gabriel Durkin that rotationally invariant states were studied recently in his $\mathrm{PhD}$ thesis [15]. We thank him for sending us his thesis. This work

was partially supported by the Polish State Committee for Scientific Research Grant Informatyka $i$ inżynieria kwantowa No PBZ-Min-008/P03/03.

\section{References}

[1] M. A. Nielsen and I. L. Chuang, Quantum computation and quantum information, Cambridge University Press, Cambridge, 2000.

[2] R.F. Werner, Phys. Rev. A 40, 4277 (1989).

[3] M. Horodecki and P. Horodecki, Phys. Rev. A 59, 4206 (1999).

[4] K.G.H. Vollbrecht and R.F. Werner, Phys. Rev. A 64, 062307 (2001).

[5] J. Schliemann, Phys. Rev. A 68, 012309 (2003).

[6] J. Schliemann, Phys. Rev. A 72, 012307 (2005).

[7] H.-P. Breuer, Phys. Rev. A 71, 062330 (2005).

[8] H.-P. Breuer, J. Phys. A: Math. Gen. 38, 9019 (2005).

[9] SU(2)-invariant states were also studied by B. Hendriks and R. F. Werner [B. Hendriks, Diploma thesis, University of Braunschweig, Germany, 2002].

[10] A. Osterloh, L. Amico, G. Falci, and R. Fazio, Nature 416, 608 (2002).

[11] T. J. Osborne and M. A. Nielsen, Phys. Rev. A 66, 032110 (2002).

[12] F. Verstraete, M. Popp, and J. I. Cirac, Phys. Rev. Lett. 92, 027901 (2004).

[13] W. Dür, L. Hartmann, M. Hein, M. Lewenstein and H. J. Briegel, Phys. Rev. Lett. 94, 097203 (2005).

[14] G.A. Durkin, C. Simon, J. Eisert and D. Bouwmeester, Phys. Rev. A 70, 062305 (2004).

[15] G.A. Durkin, Light and Spin Entanglement, PhD thesis, Oxford, 2004.

[16] T. Eggeling and R.F. Werner, Phys. Rev. A 63, 042111 (2001).

[17] T. Eggeling, On Multipartite Symmetric States in Quantum Information Theory, PhD thesis, Braunschweig, 2003 (available on line at www.biblio.tubs.de/ediss/data/20030602b/20030602b.pdf).

[18] D. Chruściński and A. Kossakowski, Phys. Rev. A 73, 062314 (2006).

[19] D. Chruściński and A. Kossakowski, Phys. Rev. A 73, 062315 (2006). 
[20] E.P. Wigner, Group Theory and its Application to the Quantum Mechanics of Atomic Spectra, (Academic Press, New York, 1959).

[21] A.R. Edmonds, Angular Momentum in Quantum Mechanics, (Princeton University Press, Princeton, 1957).

[22] L.C. Biedenharn and J.D. Louck, Angular Momentum in Quantum Physics: Theory and Applications, (Addison-Wesley Publishing Company Reading, Massachusetts, 1981).

[23] B. Terhal and P. Horodecki, Phys. Rev. A 61, 040301 (2000).

[24] A. Jamiołkowski, Rep. Math. Phys. 3, 275 (1972). 